\title{
Restauración del Pucara de Quitor
}

EduARdo MuÑoz ${ }^{1}$

\section{Introducción}

El Pucara de Quitor, ubicado a $3 \mathrm{~km}$ en dirección nororiente del pueblo de San Pedro de Atacama, es un conjunto monumental prehistórico, cuya importancia es conocida por sus características excepcionales con respecto a sus similares en el área atacameña.

El presente trabajo trata de las obras de restauración que se efectuaron entre los meses de septiembre y diciembre de 1981. El planeamiento y ejecución de la primera etapa de restauraciones en Quitor concluyó en valiosas experiencias sobre la dimensión espacial y características técnicas del habitar de los atacameños que requirió además la necesidad de la fortificación.

\section{Antecedentes}

La primera referencia escrita sobre Quitor pertenece al cronista Gerónimo de Bibar (1558), quien participó en la expedición de Pedro de Valdivia a Chile en 1540. Este cronista cuenta que al paso de los adelantados por el oasis de San Pedro de Atacama se produjo un enfrentamiento con los indígenas que se hicieron fuertes en el Pucara. Tras una dura contienda, Francisco de Aguirre al mando de 30 soldados españoles y yanaconas hicieron capitular el sitio que defendían unos 1000 indígenas atacameños. El castigo ejemplar impuesto por los españoles a los caciques capturados y que consistió en su decapitación, dio origen a que a este lugar se le diera posteriormente el nombre de "Pueblo de las Cabezas".

Los estudiosos del Pucara de Quitor coinciden en que la construcción de esta fortaleza se originó durante el Período Agroalfarero Tardío, es decir, alrededor de 1300 DC (s. XIV). La investigación arqueológica efectuada durante la ejecución de este

1 Instituto de Investigaciones Arqueológicas y Restauración Monumental, Facultad de Educación y Ciencias Humanas, Universidad de Antofagasta. Casilla 1240, Antofagasta, CHILE. proyecto verificó lo anterior, basándose para ello en las evidencias de cerámica regional y de origen altiplánico encontradas en las excavaciones.

Las investigaciones arqueológicas que se han realizado en San Pedro de Atacama desde hace años son conducentes a establecer que durante la fase tardía del Período Agroalfarero las comunidades que poblaban la zona precordillerana que circunda la Puna de los Andes Meridionales alcanzaron un evidente desarrollo cultural, económico social y por lo tanto demográfico que les permitió mantener una relativa hegemonía en esta sensible área subandina. Sin embargo, esta misma razón animó a grupos del altiplano (de la actual Bolivia) a hostilizar estas comunidades en procura de tierras cultivables (maíz), causa que al parecer obligó la concentración de la población en pueblos que fueron concebidos con fines bélicos y fortificados.

De lo anterior se deduce la presencia de pucaras casi contemporáneos dispersos en el área que circunda los Andes que va desde la sierra del sur de Perú, Tarapacá, área atacameña y Noroeste Argentino en las cotas de 2000 a 4000 m.snm. Son conocidos los pucaras de Humallani, Taltape, Copaquilla, Lupica, Belén, Saxamar, Purisa y Antara en la sierra tarapaqueña; Lasana, Chiu Chiu, Turi, Cupo, Quitor en el área atacameña y Rinconada, Tilcara, Sorcuyo, Pucara Morado, Pucara la Cueva, Humahuaca etc. en el Noroeste Argentino, por nombrar algunos (Núñez et al. 1975).

Las estructuras habitacionales del Período Agroalfarero Tardío que se han registrado en el Salar de Atacama se caracterizan por constituir pequeños aglutinamientos aldeanos ubicados principalmente en las quebradas y ríos que desembocan en el Salar. El Pucara de Quitor sirvió, en caso de peligro, para concentrar a esta población dispersa aunque también mantuvo una población estable.

De los restos arqueológicos se puede deducir que las actividades principales para la subsistencia fueron 
la recolección de chañar y algarrobo y en menor grado la agricultura (maíz) y el pastoreo (llamas, alpacas). Realizaron además otras actividades como cerámica, metalurgia en cobre, textiles, cestería, artesanía en madera y piedra, lo que demuestra un alto nivel tecnológico y una organización social y económica avanzada para su época.

La introducción de nuevos rasgos culturales nos indican que el Pucara fue ocupado durante el Período Incaico, conservándose la mayoría de los rasgos atacameños antes mencionados. Después de la ocupación incaica hay evidencias de su utilización durante el Período Colonial Temprano (s. XVI), corroborado esto último por cronistas de la época (Núñez 1982 Ms).

\section{El proyecto}

\section{Descripción del pucara}

El principal propósito del Pucara de Quitor, además del habitar, fue la fortificación con fines de defensa ante eventuales asedios bélicos. Este factor condicionó desde la elección del sitio del emplazamiento hasta el carácter de las edificaciones y la vialidad interna.

La posición topográfica elegida para construir el pucara fue excelente desde el punto de vista estratégico considerando que se ubica en la ladera sur de la quebrada por cuyo cauce fluye el río San Pedro. La erosión que afectó esta ladera originó escarpados farellones de roca al costado norte y oeste; al sur, una quebradilla abrupta enmarca la inaccesibilidad del terreno; el costado este permite el acceso por una pendiente relativamente regular, pero de una agudeza promedio de $30^{\circ}$ de ángulo de inclinación. La altura máxima del cerro desde el lecho del río alcanza $80 \mathrm{~m}$.

El espacio en que se distribuyen las construcciones abarca unos $24000 \mathrm{~m}^{2}$ con longitudes extremas de $200 \mathrm{~m}$ de largo por $120 \mathrm{~m}$ de ancho. En el acceso por el costado este (el más vulnerable) se encuentra un colosal muro de defensa dispuesto en forma serpenteante y quebrada, cubriendo prácticamente a lo ancho el pucara en una longitud aproximada de $121 \mathrm{~m}$. Su espesor es de $1.20 \mathrm{~m}$ (promedio) y su altura variable alcanza por el exterior hasta $\operatorname{los} 3 \mathrm{~m}$. La única entrada posible detectada hasta el momento para trasponer este muro son dos escalerillas que forman parte del mismo, las que bien disimuladas permiten un acceso muy controlado.
Ya en el interior, se destacan las primeras construcciones por su tamaño y forma que guardan cierta ortogonalidad. Esto se debe a que en la primera parte la pendiente es menos aguda, lo que permitió una fácil adecuación al terreno, en los niveles superiores fueron indispensables las obras de compensación y contención antes de construir. $\mathrm{Al}$ ascender podemos observar tres depresiones en el cerro que se pueden denominar como quebradillas, en ellas encontramos un tipo de estructura que se adapta a este accidente presentando efecto de cuña contra el cerro, logrado por una pared recta al frontis y otra en forma curva que completa el perímetro. El resto de las construcciones toma diversas formas según lo permite el terreno, lo que va definiendo verdaderos "barrios" de acuerdo a su ubicación en el relieve y a las necesidades de circulación. Estas condicionantes aumentan el predominio de formas curvas y mixtas en las plantas donde se conjugan tenazmente ángulos de esquina y paredes circulares. En una operación general se puede concluir una mayor frecuencia del semicírculo en las estructuras, dispuestas en un aterrazamiento irregular pero bastante efectivo que podría semejarse a las graderías de un colosal anfiteatro.

El patrón habitacional, por lo general, consiste en estructuras uniespaciales que en algunos casos están divididas en dos. En la mayoría de ellas está incluida una estructura más pequeña utilizada, seguramente, como silo. Advertimos que existe muy poca semejanza en la forma de las diversas estructuras. Se destaca en el centro del pucara una construcción semicircular de gran tamaño y que ha sido llamada "torreón" por su ligera semejanza con este elemento de los castillos medioevales europeos. Este "torreón", que probablemente fue el principal puesto de vigilancia, impresiona por su excelente ubicación con una visibilidad hasta de unos $80 \mathrm{~km}$. En la cima del cerro se encuentran estructuras aisladas y de forma rectangular. Una de ellas de gran tamaño, lo que hace suponer diferente época y función al resto de las construcciones.

La altura de los muros es bastante variable. Las evidencias nos permiten aseverar que estas son equivalentes al tamaño de los recintos. La técnica de albañilería empleada favoreció un amplio desarrollo de alturas, además la disposición en aterrazamiento y la utilización de paredes posteriores como contención de terrenos compensados contribuyeron a fundar estos paramentos en altura. En los niveles superiores ayudó a la estructuración de paredes altas la solución en curva del encuentro de muros. 
La mayor altura encontrada alcanza a $3.30 \mathrm{~m} \mathrm{si}$ se considera que fue utilizado como base el muro posterior de las estructuras del nivel inferior. La altura máxima en los interiores de habitación midió $2.20 \mathrm{~m}$, evidenciado esto por agujeros en la pared que hacen suponer vigas de techumbres.

La técnica de construcción es de un éxito notable, resultado de una favorable combinación de factores. El cerro donde se ubica el pucara está conformado principalmente por una especie de gneiss en estado de descomposición de color blanco grisáceo. Esta roca era apta para los fines constructivos y se encontraba en gran cantidad en el mismo sitio, su extracción y trozadura es relativamente fácil.

Como mortero se uso argamasa de arcilla que extrajeron de las laderas del río, cercanas al pucara. La calidad de la argamasa, en este caso, se debe exclusivamente al grado de salinidad de la arcilla, lo que le aporta un poder de cohesión y endurecimiento excepcional ( $\sin$ necesidad de mezclarla con otros elementos). Sin embargo, esta cualidad no sería tal en un ambiente que no reúna condiciones de sequedad extrema como lo es en el desierto atacameño. Las sales que componen principalmente estas arcillas salinas son el cloruro de sodio y el bórax.

Las albañilerías son bastante heterogéneas. Las hay de diferentes calidades en cuanto a la técnica de fundaciones, traba de hiladas de encastillamiento y el empleo del mortero de pega. En muchos casos el uso de este último elemento es bastante moderado debido al difícil transporte por las agudas pendientes del cerro del pucara. A pesar de lo anterior, se puede calificar como un buen desarrollo de la técnica. Se escogió el tamaño de la piedra en cada situación y el sentido de la colocación, en las hiladas hay alternancia de la traba en sentido horizontal y vertical, en las bases de contención hay verdaderos "pilares" que consisten en la colocación vertical de una piedra de gran tamaño y forma alargada que prácticamente aprisiona el muro al suelo. Las piedras utilizadas en la construcción de los muros no muestran señas de haber sido canteadas, sólo hay evidencias de extracción, trozadura, elección y colocación adecuada de la forma resultante.

Los vanos de puertas fueron solucionados mediante la colocación de piedras largas a manera de jambas. Entre las ruinas no se identificaron dinteles, lo que hace presumir que hubiesen sido de madera, y estos son reutilizados inmediatamente después del abandono. Sin embargo, Latcham (1938) afirma haber visto dos dinteles de piedra durante su visita al pucara. Aunque las techumbres han desaparecido en su totalidad, podemos suponer su factura en maderos sin elaborar de árboles existentes en el oasis (algarrobo, tamarugo), usados como vigas, la cubierta de ramas, paja brava y barro.

El Pucara de Quitor, con aproximadamente 164 estructuras construidas, puede considerarse que logró en buena forma adaptación y coherencia con su paisaje, como también la efectividad para el habitar y el defender.

Es necesario destacar que entre las expresiones arquitectónicas de los atacameños, los pucaras son los máximos exponentes. En ellos ya se manifiesta el sentido plástico de los volúmenes unido a la funcionalidad, con voluntad de aparecer distintos a las obras que les precedieron. La época de los pucaras se caracteriza además por ser la culminación de las tecnologías constructivas que evolucionaron en la zona desde tiempos milenarios.

\section{Cuantificación del estado de ruina}

El estado en que se encontró el pucara, al iniciar los primeros estudios para el proyecto en el mes de julio de 1981, era principalmente una confusión de volúmenes indefinidos, debido a la irregularidad de las formas de los muros afectados masivamente por los derrumbes. Se sumaban a esto los escombros de los mismos diseminados en torno a ellos. Este inconveniente dificultaba la apreciación del conjunto considerando además un mimetismo por color y forma de las piedras de las construcciones provenientes del mismo cerro.

Al examinar el interior de la fortaleza se observó el avanzado estado de ruinas que impedía vislumbrar las vías de acceso a las diferentes estructuras y niveles, incluso las formas de muchas de ellas eran imperceptibles por estar sepultadas con los escombros de sus propios muros.

Las causas principales del estado de ruina eran el abandono por casi 400 años a la acción de los elementos como lluvia, viento, sismo y la reutilización de sus materiales en otras construcciones en sus proximidades, sin conocerse en este lapso de tiempo ningún tipo de trabajo de conservación. Otra causa también que ha influido en este deterioro sobre todo en los últimos 10 años es la afluencia de visitantes 
que en forma desordenada circulan a través de los muros afectados por derrumbes, aumentando considerablemente los daños.

En un análisis detenido se pudo establecer el avance del estado de ruinas en un 50\%. Teniendo en cuenta la antigüedad de la construcción, casi 700 años, más los factores de destrucción, se puede concluir que la técnica constructiva empleada es adecuada y por lo tanto el estado actual es producto de los agentes mencionados.

\section{Formulación del tipo y técnica de restauración}

La importancia del Pucara de Quitor como testigo material de la prehistoria ejemplifica aspectos que se relacionan con la particular cosmogonía atacameña inserta en un ambiente geográfico de desierto marginal, circunscrito en una compleja área de influencia panandina. Lo anterior condicionó la formulación de un proyecto de conservación y restauración que contempló lo siguiente:

1) Conservación fidedigna de los restos culturales allí implícitos susceptibles de ser interpretados en forma científica.

2) Congelación del estado de ruina por medio de la consolidación de paramentos, lo que produciría fundamentalmente la restitución de las unidades estructurales afectadas.

3) Liberación de volúmenes que se presentaban ilegibles por estar sus nexos saturados por sus propios escombros.

4) Planteamiento de hipótesis, a partir de la interpretación de las ruinas, sobre las características predominantes de la reconstrucción de volúmenes fijando el marco hipotético que la limitara y detuviera ante el surgimiento de dudas respecto a las formas originales.

5) Valorización general de los volúmenes a fin de facilitar la comprensión del conjunto.

Los criterios generales adoptados en este proyecto se delinearon a partir de las Normas Internacionales sobre conservación y restauración monumental, sancionadas por UNESCO.

\section{Las obras}

\section{Cartografía y topografía}

La primera actividad en terreno fue el levantamiento taquimétrico del cerro del pucara. Esto se cumplió a completa satisfacción de las necesidades del proyecto, por tres geomensores de la Universidad de Antofagasta. El resultado obtenido fue el plano del relieve en curvas equidistantes a $1 \mathrm{~m}$ (Figura 1).

El dato anterior permitió desarrollar la cartografía completa de lo restaurado, corrigiendo el plano base con que se contaba de C. J. Montt (Mostny 1949). De este plano base se conservó la numeración de las estructuras.

\section{Montaje de obras}

En septiembre de 1981 se inició el montaje de obras para la restauración del Pucara de Quitor. Prioritarios fueron los trabajos de habilitación de un acceso expedito al cerro del pucara a fin de movilizar materiales y equipos para la obra (Figura 2). Esto significó la construcción, con piedras y arcilla, de un tramo de $300 \mathrm{~m}$ de camino a través de arenales, aprovechando un antiguo trazado existente. Solucionado el acceso, se planteó la solución para elevar los materiales y equipos por las agudas pendientes del cerro. Se construyó en la quebrada del costado sur un puente de madera equipado con roldana para izar baldes con arcilla a una altura de $5 \mathrm{~m}$. Se habilitó un sendero de pendiente moderada para carretillas de mano, por la ladera hasta una cancha de materiales que se instaló en las construcciones del primer nivel ( $8 \mathrm{~m}$ de altura). Paralelamente a esta labor, se instaló una motobomba de 12.5 HP para elevar una columna de agua de 2" hasta la cancha del primer nivel. El agua se almacenó en 10 tambores de 2001 cada uno.

Las arcillas que se emplearon se colectaron en el ayllu de Solor, distante $8 \mathrm{~km}$ del pucara, por ser estas de mejor calidad que las utilizadas originalmente.

Una vez establecida la cancha de materiales fue necesario movilizar la argamasa desde allí hasta las estructuras en restauración. Esta labor no fue posible aquí con el tradicional sistema de carretillas debido al terreno difícil, entonces, hubo que confeccionar angarillas de madera capaces de contener hasta 601 de material que era transportado por dos hombres. 


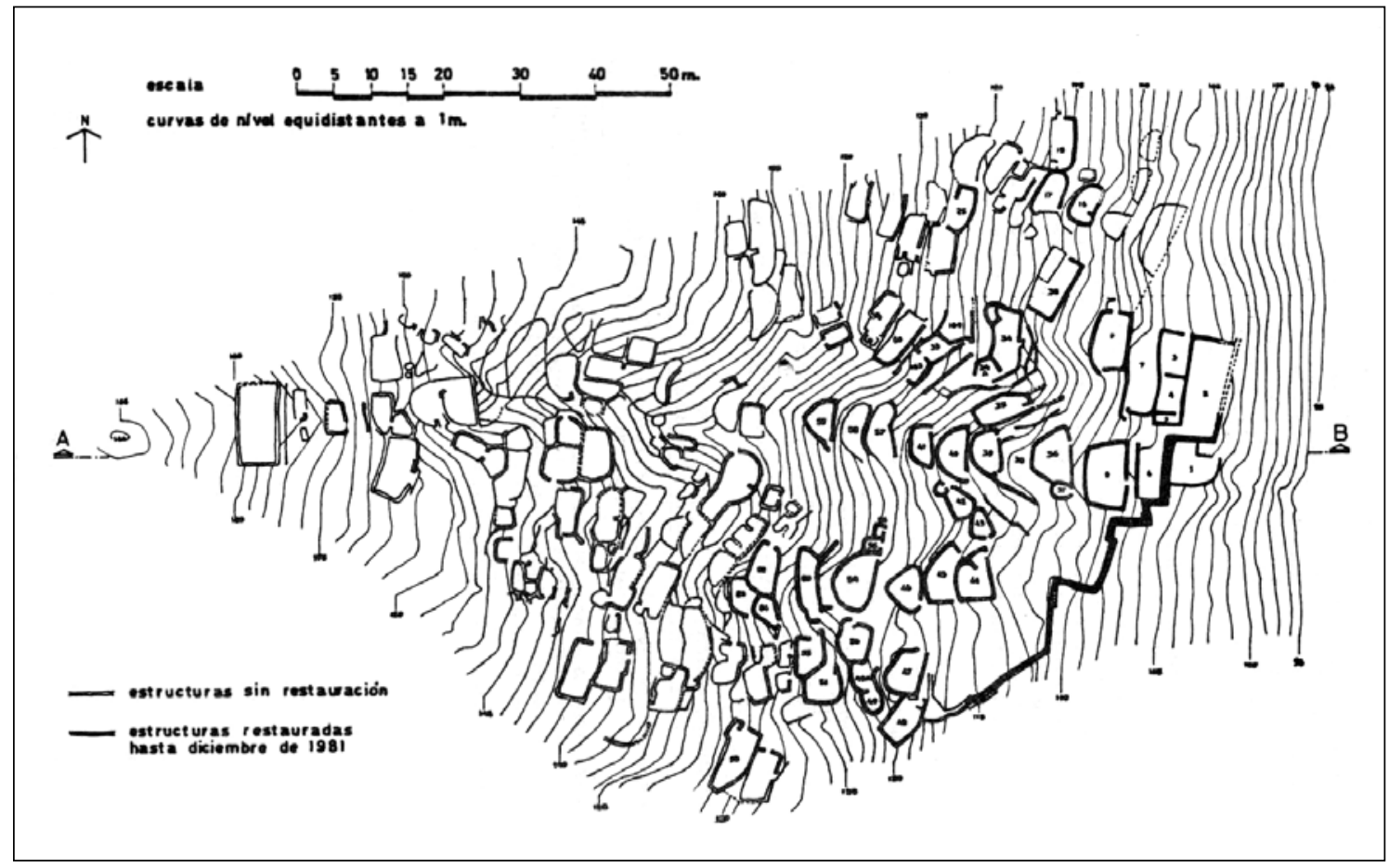

Figura 1. Restauración del Pucara de Quitor. Planta del conjunto.

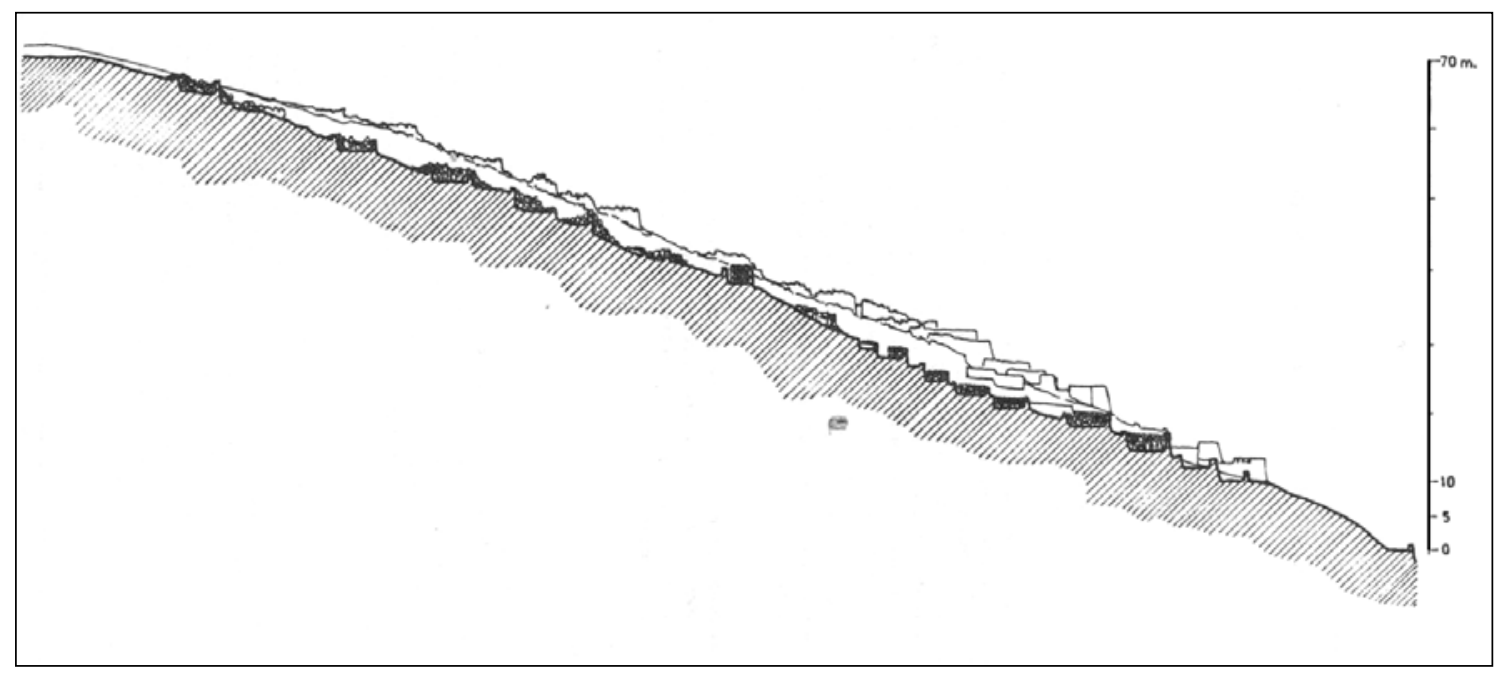

Figura 2. Restauración del Pucara de Quitor. Corte A-B. 
Transcurridas las primeras jornadas de restauración en el primer nivel establecido, la cancha de materiales se hizo inoperante, por la lejanía y las pendientes agudas que debían sortearse antes de llegar a los lugares de restauración. Este inconveniente obligó a idear y construir un andarivel de $16 \mathrm{~m}$ de largo con una inclinación de $35^{\circ}$; la solución permitió elevar las arcillas hasta la cota de los $20 \mathrm{~m}$ de altura, pudiéndose allí establecer una nueva cancha. El agua se hizo subir prolongando la cañería de 2" hasta este último nivel. Conjuntamente a las labores mencionadas, se restauraron vías de comunicación, y se suplieron terrenos difíciles con carreras de madera, esta operación hizo que se desplazaran en forma expedita los materiales, lo que significó cubrir una amplia extensión afecta a restauración.

Al plantearse una segunda etapa debido al refinanciamiento del proyecto, las instalaciones antes descritas resultaron insuficientes para continuar trabajando en los niveles superiores. Esto obligó a diseñar y construir un segundo andarivel de $22 \mathrm{~m}$ de longitud, con $40^{\circ}$ de inclinación, de características similares al primero con sistema de poleas y torno, por el cual se transportaron las arcillas hasta la cota de $35 \mathrm{~m}$ de altura. El agua se hizo llegar a este nivel, reduciendo la columna de 2" a 1 y $1 / 2$ ". Estos obrajes tuvieron que ser completados con la habilitación de nuevos senderos, construcción de pasarelas y carreras para conseguir el desplazamiento de materiales por terrenos mucho más accidentados que los de los niveles inferiores.

A medida que avanzó la restauración, la cantidad de piedras a reutilizar disminuyó notablemente, la causa que se detectó fue la ocupación de este material en pircados de las chacras contiguas al pucara. Para suplir la escasez se habilitó una cantera en la quebrada sur en donde se encontró el mismo material pétreo usado originalmente, se extrajeron alrededor de 100 toneladas. Para la segunda etapa se construyó un puente para izar las piedras a la cota de los $25 \mathrm{~m}$ de altura, logrando un desplazamiento y distribución eficaz.

La arcilla, antes de transformarse en argamasa de pega llegó a cumplir un recorrido de $100 \mathrm{~m}$ de distancia y a una altura de $35 \mathrm{~m}$ desde el lugar de descarga. La columna de agua se bombeó hasta la altura de $40 \mathrm{~m}$ desde el lugar de captación.
La restauración

El criterio general adoptado, definió el tipo de restauración, referido previamente. Este tipo contó con las siguientes actividades:

Consolidación. Las investigaciones previas a las obras demostraron que las causas de la ruina de las construcciones y su potencial progresión se debían principalmente a la pérdida del equilibrio estructural de los recintos afectados por zonas en derrumbe. Se resolvió la consolidación a partir de la reconstrucción de muros, devolviendo la estructuralidad perdida.

Otro factor de importancia en esta actividad fue el uso del mortero de pega. La técnica se mejoró sustancialmente al utilizar arcillas de mejor calidad y en mayor cantidad que las empleadas originalmente. Los métodos modernos que se utilizaron para la movilización del material hasta las obras permitieron disponer de mezclas más generosas. Se advirtió en muchos casos que la pobreza del mortero de pega, por su evidente dificultad de transporte, fue determinante para su mala conservación.

Los encastillamientos de la restauración observaron rigurosamente la técnica original de hiladas y trabamientos de probada eficacia.

Este método de consolidación aportó a las construcciones restauradas una resistencia superior a la lograda por sus creadores, sin necesidad de la intromisión de materiales extraños.

Recuperación de la espacialidad y vialidad interna. Al determinar que los restos yacentes de las construcciones no serían removidos sino para ser reutilizados en su propia restauración, se dificultaba la apreciación global del conjunto y la identificación de las formas originales. El valioso concurso de la técnica arqueológica para remover el material caído facilitó la certera interpretación de las evidencias y su posterior restauración fidedigna. Se identificaron correctamente las estructuras habitacionales con sus silos interiores y exteriores, patios del habitar y comunitarios, plazas de concentración militar, terrazas, terraplenes y callejones. Luego se estableció el dimensionamiento de alturas a restaurar a fin de recuperar su espacialidad.

El avance de las obras obligó a agilizar el desplazamiento por los terrenos del pucara, esta situación activó el tratamiento paralelo de la vialidad interna, 
lo que contribuyó además a liberar las estructuras facilitando el tratamiento de los volúmenes.

Valorización general. El concepto del espacio urbano regulado por la dimensión requerida por cada una de las funciones que acoge, se recuperó luego de la restauración de las estructuras y la vialidad. Sin embargo, la condición de ruinas en que se encontraba el pucara que no permitía una reconstrucción del 100\% por falta de testimonios, hacía un tanto subjetivo el manejo de los límites de ésta. El criterio adoptado en este sentido exigió principalmente facilitar la mejor comprensión del conjunto. Para lograr este importante factor, se estableció una contraposición a las formas mimetizadoras entre el estado de ruinas y el cerro, mediante la imposición de la horizontal en los paramentos restaurados. Esta técnica valorizadora definió los volúmenes enmarcando la espacialidad del conjunto consiguiendo además visualizar la importancia monumental del sitio.

Resultado general. De las 164 estructuras se restauraron 45 de ellas con sus terrazas, terraplenes y vías de circulación. Además, se reconstruyeron $91 \mathrm{~m} \mathrm{del}$ muro de defensa con sus escalerillas de acceso. La zona restaurada abarca una superficie de $5500 \mathrm{~m}^{2}$. La ponderación del grueso de la obra avanzada se estima en un $35 \%$ del total del pucara.

\section{Conclusiones}

La complejidad de las obras de restauración monumental impide establecer pautas o fórmulas para imponer determinadas técnicas. Cada monumento debe ser objeto por separado de una intensa investigación y contar con el concurso de los especialistas que sean necesarios para proyectar su conservación y restauración. Sí, en cambio, debe primar un criterio general, para ello hay que acudir a las Normas Internacionales sobre Conservación y Restauración que han sido elaboradas por expertos de todo el mundo. En ellas está contenido el rigor de la disciplina que se ha ido plasmando a través de una larga experiencia.

La restauración del Pucara de Quitor arrojó nuevas experiencias en cuanto al desarrollo de técnicas especiales por las características accidentadas del terreno en que se trabajó. Estas técnicas consiguieron la movilización de materiales y equipos desafiando un terreno hostil. La investigación de las causas del estado de ruinas, de la técnica de los morteros de pega, y los sistemas de encastillamiento fueron resultantes de una eficaz consolidación que puede asegurar la pervivencia del monumento por muchos años más. El concurso permanente de la disciplina arqueológica aportó la definición de la naturaleza de las formas para su reconstrucción y además consiguió datos valiosísimos para identificar, ubicar en el tiempo y relacionar este rasgo cultural atacameño con el contexto andino.

\section{Participantes en el Proyecto}

Eduardo Muñoz, restaurador, investigador responsable del proyecto; Patricio Núñez, arqueólogo, coautor del proyecto general; Jaime Valenzuela, restaurador, coautor del proyecto de restauración; Roberto Montandón, experto del Consejo de Monumentos Nacionales, asesoría en el proyecto; Manuel Ayala, Mario Briceño, Jorge Hewstone, ingenieros en geomensura, autores del levantamiento taquimétrico del cerro del pucara.

\section{REFERENCIAS CITADAS}

BIBAR, G. DE 1966. Crónica y relación copiosa y verdadera de los reynos de Chile por Gerónimo Bibar, natural de Burgos 1558. Fondo Histórico Bibliográfico José Toribio Medina, Santiago.

LATCHAM, R., 1938. Arqueología de la región atacameña. Universidad de Chile, Santiago.

MONTANDON, R., 1950. Apuntes sobre el Pucara de Lasana. Cuadernos del Consejo de Monumentos Nacionales 1.
MOSTNY, G., 1948-49. Ciudades atacameñas. Boletín del Museo Nacional de Historia Natural XXIV.

NUÑEZ, L., V. ZLATAR y P. NUÑEZ, 1975. Relaciones prehistóricas trasandinas entre el Noroeste Argentino y el norte Chileno (Período Cerámico). Serie Documentos de Trabajo 6, Universidad de Chile, Antofagasta.

NUÑEZ, P., 1982Ms. Significado del Pucara de Quitor. 